Research Paper

\title{
Survival changes in Patients with Synovial Sarcoma, 1983-2012
}

\author{
Shuncong Wang $1^{*}$, Rongfeng Song ${ }^{*}$, Tiantian Sun ${ }^{3}$, Bingzong Hou ${ }^{4}$, Guobin Hong5, Saradhi Mallampati6, \\ Hongliu Sun ${ }^{7}$, Xiuling Zhou1 ${ }^{1}$, Cuiling Zhou ${ }^{1}$, Hongyu Zhang ${ }^{1}$, Zhibin Cheng' ${ }^{1}$, Jie Li ${ }^{8}$, Haiqing Ma1 ${ }^{\bowtie}$, \\ Huanhuan Sun ${ }^{1 凶}$ \\ 1. Department of Oncology, The Fifth Affiliated Hospital of Sun Yat-sen University, Zhuhai, Guangdong 519000, China; \\ 2. Department of Gastroenterology, Cancer Hospital of Jiangxi Province, Nanchang, Jiangxi 330029, China; \\ 3. Department of Hematology, The First Affiliated Hospital of Sun Yat-sen University, Guangzhou, Guangdong 510080, China; \\ 4. Department of Gastrointestinal Surgery, The Fifth Affiliated Hospital of Sun Yat-sen University, Zhuhai, Guangdong 519000, China; \\ 5. Department of Radiology, The Fifth Affiliated Hospital of Sun Yat-sen University, Zhuhai, Guangdong 519000, China; \\ 6. Department of Laboratory Medicine and the Center for Stem Cell and Developmental Biology, The University of Texas MD Anderson Cancer Center, \\ Houston, TX 77030, USA; \\ 7. Department of pathology, University of Michigan, Ann Arbor, MI 48201, USA; \\ 8. Department of Breast and Thyroid Surgery, The First Affiliated Hospital of Sun Yat-sen University, Guangzhou, Guangdong 510080, China.
}

*These authors contributed equally to this work.

$\triangle$ Corresponding author: Haiqing Ma, M.D. \& Ph.D., Department of Oncology, The Fifth Affiliated Hospital of Sun Yat-sen University, Zhuhai, Guangdong 519000, China; (mahaiqing@mail.sysu.edu.cn) Work Telephone: +86-0756-2528002; FAX: +86-0756-2528210 or Huanhuan Sun, M.D. \& Ph.D., Department of Oncology, The Fifth Affiliated Hospital of Sun Yat-sen University, Zhuhai, Guangdong 519000, China; (huanhuansun10@163.com) Work Telephone: +86-0756-2528888; FAX: +86-0756-2528210

(C) Ivyspring International Publisher. This is an open access article distributed under the terms of the Creative Commons Attribution (CC BY-NC) license (https://creativecommons.org/licenses/by-nc/4.0/). See http://ivyspring.com/terms for full terms and conditions.

Received: 2016.08.25; Accepted: 2017.05.02; Published: 2017.07.01

\begin{abstract}
Background: Synovial sarcoma (SyS) is a rare malignancy that typically invades the extremities and occurs predominantly in adolescents. Studies on incidence and survival in SyS that were based on a large population had not been reported yet.

Methods: To evaluate changes in incidence and survival in SyS over three decades, we accessed data on SyS cases in each decade between 1983 and 2012 (1983-1992, 1993-2002, and 2003-2012) from the Surveillance, Epidemiology, and End Results (SEER) database. The survival difference between decades, age groups, sexes, race, pathologic types, sites, stages and socioeconomic status (SES) over three decades were accessed by comparing Kaplan-Meier curves.

Results: We located 2,070 SyS cases in 18 SEER registry regions between 1983 and 2012. Our study demonstrated that the incidence of SyS per 1,000,000 continued to increase from 0.906 to 1.348 to 1.548 in the total population and in most age groups and that the age of incidence peak was 15-29 years in three decades. But, the survival of patients with SyS did not significantly improve throughout the three decades, with 5 -year survival rates of $69.4 \%, 61.1 \%$ and $60.5 \%$ respectively $(p$ $>0.05$ ). Interestingly, the widening survival gaps among races, sexes, pathological types and various SES over time were observed, with narrowing $p$ values.

Conclusions: This study demonstrated the increasing incidence and unimproved survival rates across three decades in a large sample, indicating the urgency for further development of diagnosis, improving health care providers' awareness of SyS and lead to the development of novel treatments.
\end{abstract}

Key words: synovial sarcoma, incidence, survival, sites and stages

\section{Introduction}

Synovial sarcoma (SyS) is an uncommon malignancy that predominately occurs in adolescents and young adults, accounting for $5 \%-10 \%$ of the soft tissue sarcomas [1]. SyS typically originates in the 
extremities and lower thigh, and traditional treatments for localized SyS include surgical resection with wide margin, radiotherapy, and adjuvant chemotherapy. However, clinical outcome in this disease based on a large sample size had not been published due to its lower incidence. Previous studies mainly consisted of case reports or focused on specific patient groups such as pediatric SyS or SyS of the head and neck [2-6]. Following advances in pathological diagnosis, surgery, chemotherapy, and radiotherapy in SyS, this study aimed to clarify the incidence and survival changes between 1983 and 2012 by accessing data in the Surveillance, Epidemiology, and End Results (SEER) database. In addition, this study compared patient survival in various stratification settings and identified the factors associated with survival.

\section{Methods}

Data collection and categorized SyS cases across three decades. All data were obtained from the SEER database, maintained by the National Cancer Institute. In this study, we collected SyS incidence data across three decades from the original nine SEER sites and survival data from 18 SEER sites. SyS is histologically defined by the following International Classification of Diseases for Oncology, Third Edition, histology codes for malignant cases: synovial sarcoma, not otherwise specified (NOS) (9040/3), synovial sarcoma, spindle cell (9041/3), synovial sarcoma, epithelioid cell (9042/3), and synovial sarcoma, biphasic (9043/3). Patients were divided into five age groups: 0-14 years, 15-29 years, 30-44 years, $45-59$ years, and 60+ years. The tumor sites were categorized into five regions: head and neck, trunk, lung and pleura, extremities, and other. We analyzed patients whose SyS had been diagnosed between1983 and 2012, the period that was divided into three decades (1983-1992, 1993-2002, and 2003-2012). We stratified cases by stage and tumor site for analyzing incidence and by SES (socioeconomic status), race, sex, stage, site, and pathological type for conducting Kaplan-Meier survival analysis. The three SES groups were defined on the basis of the county poverty rate [7-9].

Incidence and survival analysis. The incidence rates of SyS were calculated per 1,000,000 persons. We compared the survival difference by Kaplan-Meier survival analysis with a two-tailed log-rank test. The survival difference between decades, age groups, sexes, race, pathologic types, sites, stages and socioeconomic status (SES) over three decades were accessed by comparing Kaplan-Meier curves.

Cox regression analyses and Spearman rank correlation analyses. Only Whites and Blacks were included in the Cox regression analysis; patients whose tumor origination site was "other" were excluded. In addition, the original high-poverty group and medium-poverty group were redefined as a new "med-high-poverty group." Ages were divided into five groups, as previously discussed. Spearman rank correlation coefficient was calculated because race was not normally distributed.

In addition, Stata MP 14 software (Stata Corp) was used for Cox regression analysis and Spearman rank correlation analysis. A two-tailed $p$ value of < 0.05 was considered statistically significant.
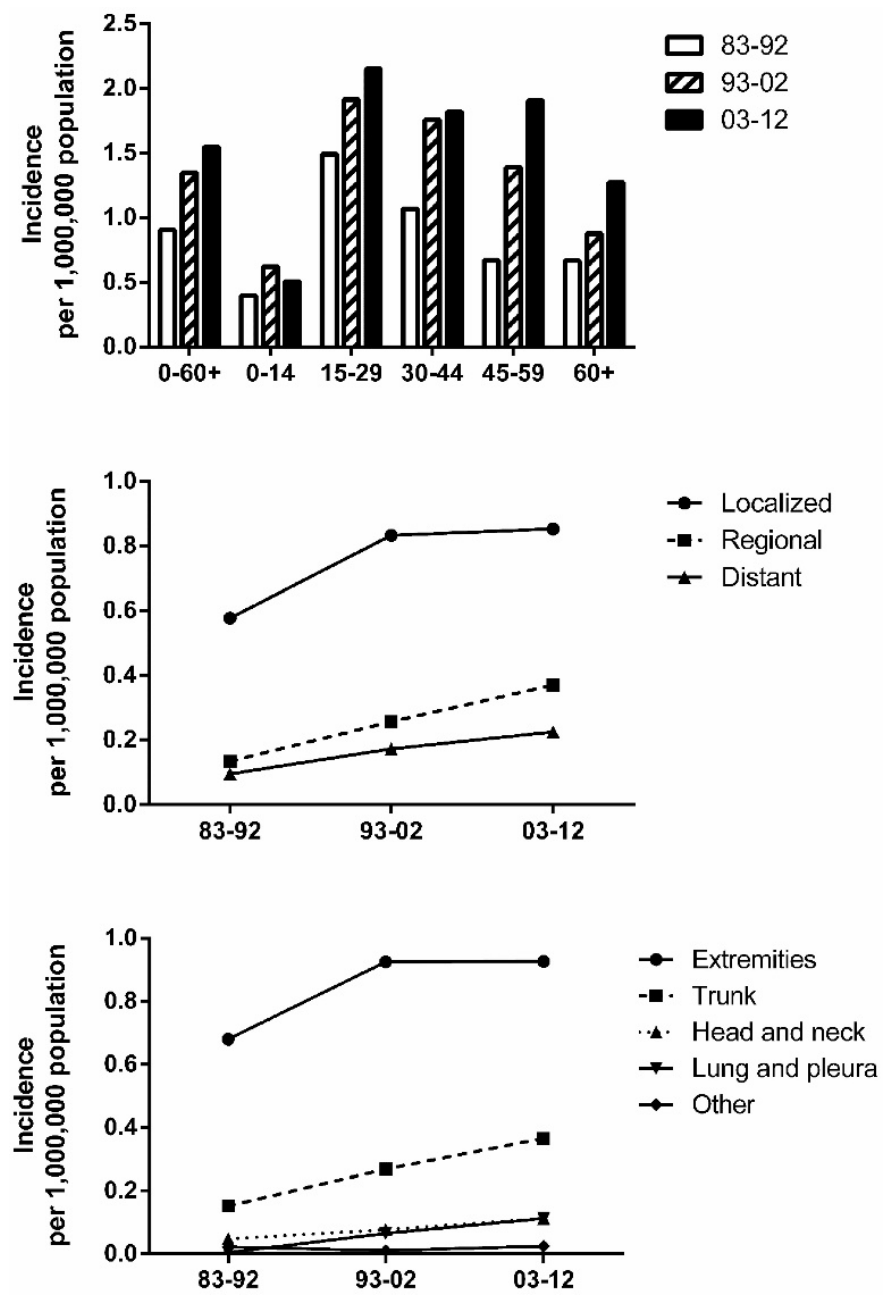

Figure 1. Summary incidences of patients diagnosed as having SyS between 1983 and 2012 at the original nine SEER sites. Incidence data were categorized by age, decade, stage, and site.

\section{Results}

Incidence trends in SyS. To maintain consistency among the registry sites surveyed and thereby improve the comparability of incidence data across the three decades, we extracted incidence data at the nine original registry sites in the SEER database, maintained by the National Cancer Institute. We 
identified a total of 999 patients diagnosed as having SyS between 1983 and 2012. As shown in Figure 1, the total incidence of SyS between 2003 and 2012 was higher $(1.548$ per $1,000,000)$ than that between 1993 and 2002 (1.348 per 1,000,000), which was higher than that between 1983 and 1992 (0.906 per 1,000,000). A similar tendency could be found in most age groups except for the 0-14 years group, in which the incidence of SyS decreased in the third decade. The SyS incidence per 1,000,000 increased substantially in the of 45-59 years group, from 0.670 in 1983-1992 to 1.392 in 1993-2002 to 1.905 in 2003-2012 (Fig. 1). The peak age of SyS occurrence was 15-29 years in the three decades, and the $45-59$ years group served as another
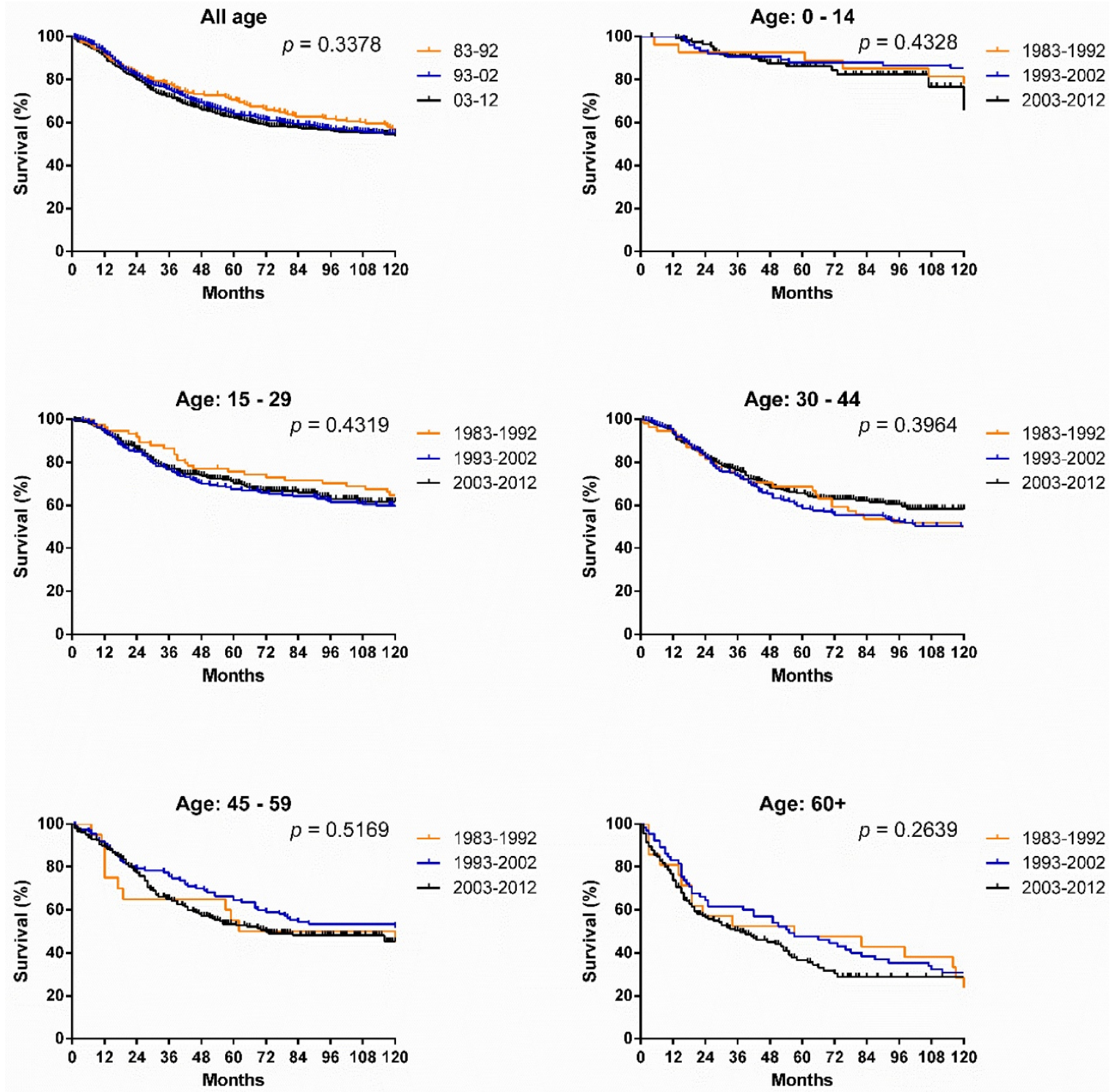

incidence peak in the third decade.

SyS occurrence by stage and by site. As illustrated in Figure 1, the incidence in various stages (localized, regional, and distant) increased over the three decades, with the highest incidence in patients with localized disease. The incidence per 1,000,000 of localized cases increased the most, from 0.577 in 1983-1992 to 0.833 in 1993-2002 to 0.853 in 2003-2012. A similar increase in incidence can be seen in the various site groups, with the incidence per 1,000,000 in the extremities, accounting for the greatest proportion of SyS cases, increasing the most, from 0.671 in $1983-1992$ to 0.925 in 1993-2002 to 0.927 in 2003-2012.

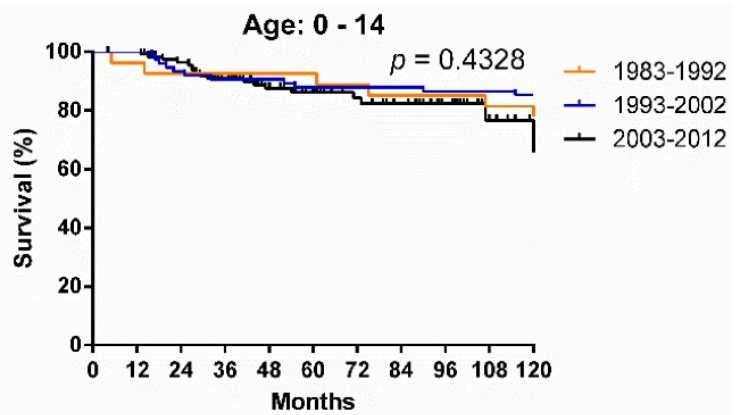

Months

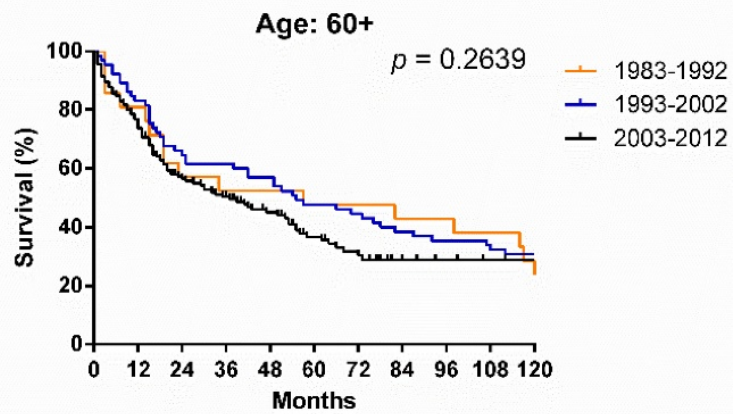

Figure 2. Kaplan-Meier survival analysis for patients with SyS at 18 SEER sites between 1983 and 2012 according to age group (total and ages 0-14, 15-29, 30-44, $45-59$, and $60+$ years) 
Survival changes in SyS. A total of 2,070 patients with SyS were identified between 1983 and 2012 at 18 registry sites in the SEER database. The median survival of patients with SyS decreased from 249 months to 199 months over the first two decades. As illustrated in Figure 2, survival in patients with SyS did not significantly improve in the total population, with 5 -year rates of $69.4 \%, 61.1 \%$ and $60.5 \%$ respectively, across the three decades. Similar tendency can be seen in each age group. Significant survival advantage in females was noted across the three decades, and the survival disparities between the sexes continued to widen each decade, with $p$ values decreasing from 0.1389 to 0.0036 to 0.0013 (Fig. 3).
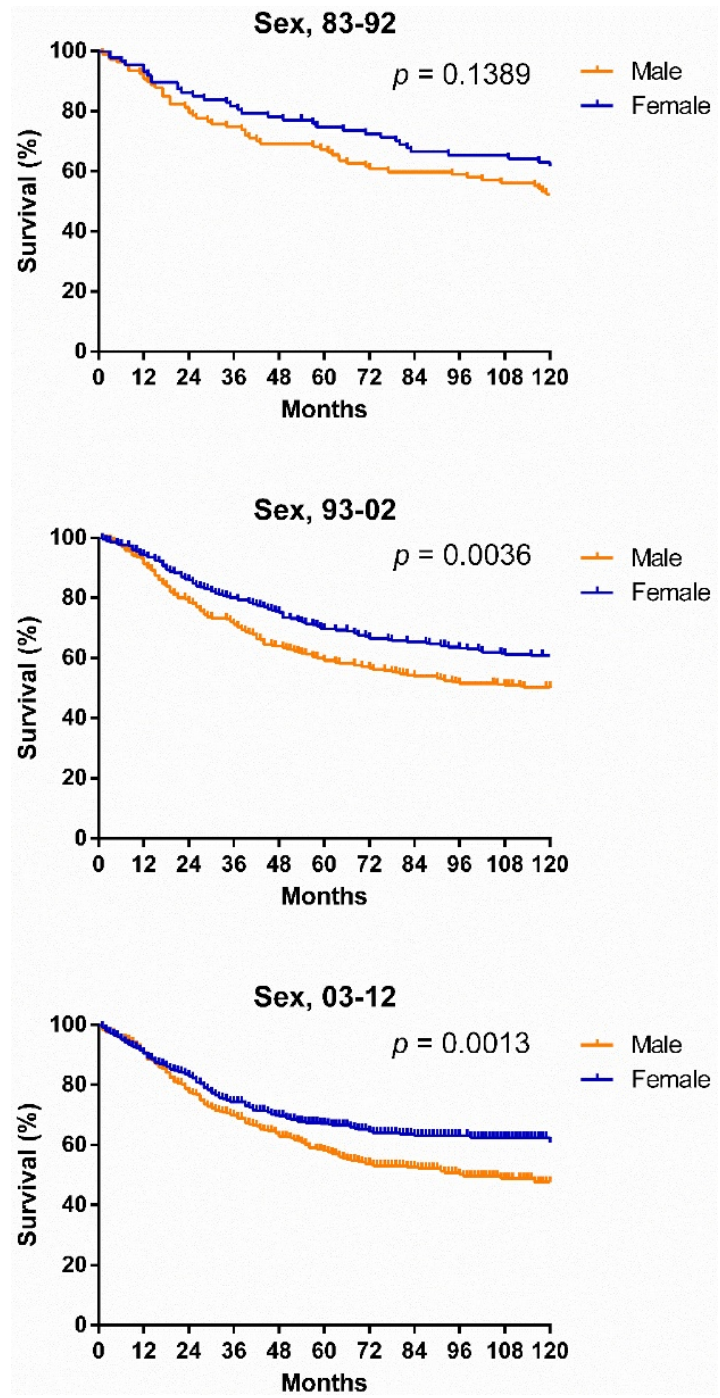

Figure 3. Kaplan-Meier survival analysis for patients with SyS at 18 SEER sites between 1983 and 2012 according to sex and calendar period.

Additionally, sex, age, race, stage, site, and pathological type were significantly associated with overall survival over the three decades according to
Cox regression analyses $(p<0.05)$ (Table 1$)$. Females, with a hazard ratio (HR) of 0.751 , were associated with a lower risk of death during the three decades, with males as the reference. In addition, older age (HR $=1.405)$, Blacks $(\mathrm{HR}=1.292)$, and advanced stage $(\mathrm{HR}$ $=2.960$ ) were associated with higher risks across the three decades, with younger age, Whites, and localized stage as references, respectively (Supplementary Table S1). When the data for each decade were analyzed, more variables showed as independent predictors in time.

Table 1. Summary data for Cox regression analysis of survival in patients with synovial sarcoma in total three decades at 18 SEER sites.

\begin{tabular}{lll}
\hline Variable & Hazard Ratio $(95 \% \mathrm{CI})$ & $p$-value \\
\hline All 1983-2012 & & \\
Univariate & $0.718(0.622-0.828)$ & $<0.001$ \\
Sex & $1.468(1.381-1.561)$ & $<0.001$ \\
Age & $1.350(1.107-1.647)$ & $=0.003$ \\
Race & $1.048(0.908-1.210)$ & $=0.519$ \\
SES & $3.091(2.819-3.388)$ & $<0.001$ \\
Stage & $1.388(1.284-1.500)$ & $<0.001$ \\
Site & $0.879(0.804-0.960)$ & $=0.004$ \\
Pathological type & & \\
Multivariate & $0.751(0.651-0.867)$ & $<0.001$ \\
Sex & $1.405(1.321-1.495)$ & $<0.001$ \\
Age & $1.292(1.059-1.577)$ & $=0.012$ \\
Race & $2.960(2.693-3.252)$ & $<0.001$ \\
Stage & $1.162(1.075-1.257)$ & $<0.001$ \\
Site & $0.900(0.823-0.985)$ & $=0.022$ \\
Pathological type & & \\
\hline Abbreviations: $95 \%$ CI $95 \%$ confidence interval; SES, socioeconomic status.
\end{tabular}

Abbreviations: 95\% CI, 95\% confidence interval; SES, socioeconomic status.

SyS survival in various races and SES groups. Whites had a higher survival rate than did Other, whose survival rate was higher than that of Blacks in the last two decades. Kaplan-Meier survival analysis showed that although no statistically significant differences between races were found, the survival gaps between races widened, with $p$ values narrowing from 0.3162 to 0.0902 to 0.0736 . In terms of SES, the longest survival was observed in the low-poverty group, with the shortest survival in the high-poverty group, and more importantly, the survival disparities among three SES groups continued to widen each decade ( $p=0.0141)$ (Fig. 4).

SyS survival by stage, by site, and by pathological type. Survival in the various stages differed significantly over the three decades and each decade analyzed $(p<0.0001)$, with the longest survival in localized cases and the shortest survival in distant cases (Fig. 5[a]). However, the survival for cases in each stage did not show significant improvement across the three decades (Fig. 6). After stratifying patients by site, the survival in the various sites differed significantly in the three decades and in each decade analyzed. In the total three decades, the 
longest survival was observed in head and neck cases, followed by extremities and trunk cases. It is noteworthy that in the last two decades, survival for head and neck cases improved dramatically (Fig. 5).

Patients with various pathological types showed significantly different survival across the three decades, and the survival gaps in each decade in the various pathological types widened in time, with narrowing $p$ values. Patients with biphasic SyS showed the longest survival in the total three decades and in the last two decades. Interestingly, the survival of patients with NOS SyS decreased in the last two decades, whereas survival of patients with biphasic and spindle cell SyS increased (Fig. 7[a]). Only survival in patients with NOS SyS decreased significantly over the three decades $(p=0.003)$; survival for the rest of the pathological types remained stable (Fig. 7[b]). a

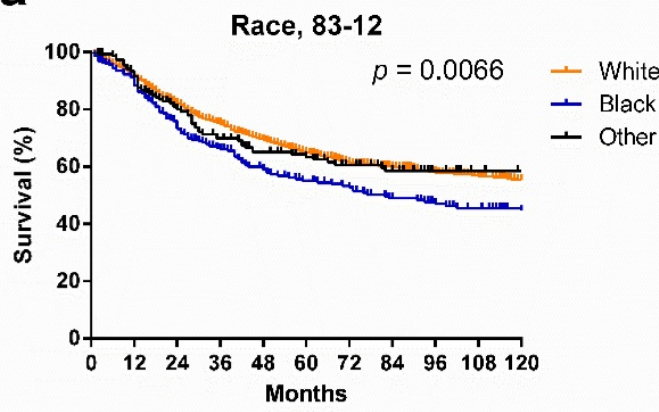

Race, 83-92

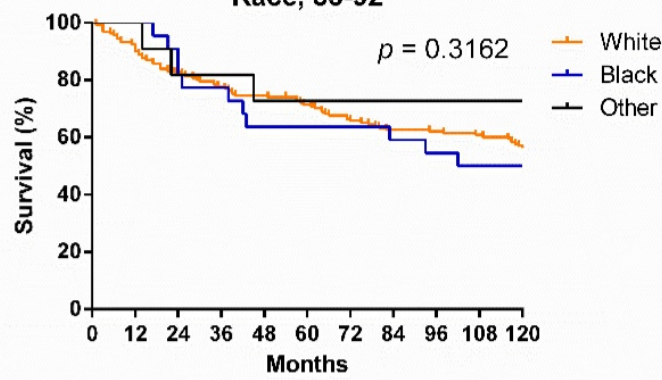

Race, 93-02

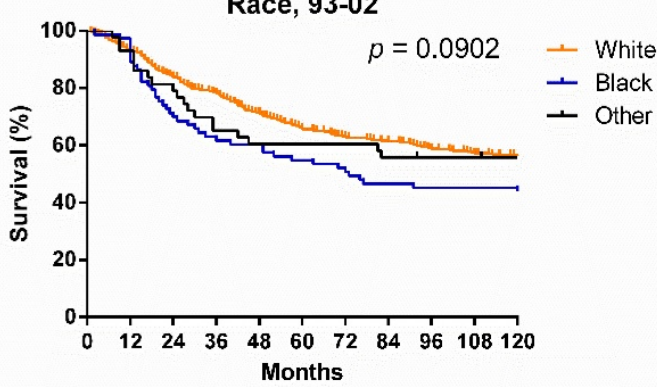

Race, 03-12

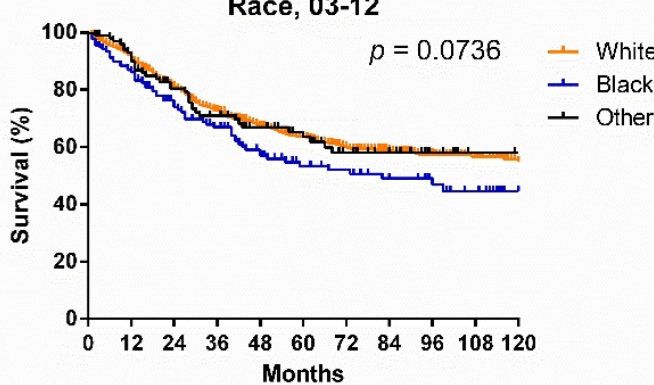

b

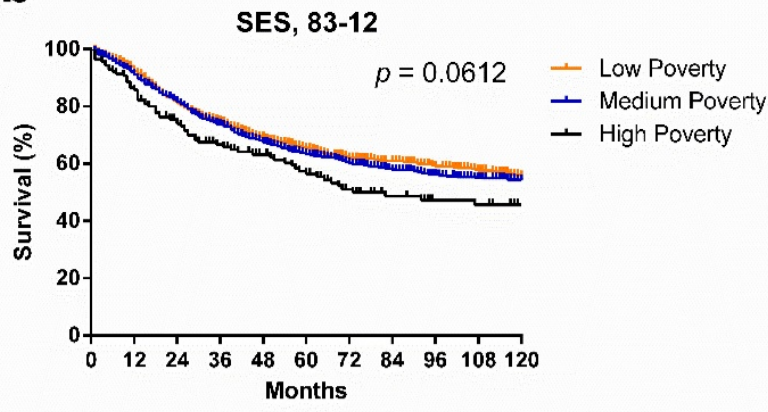

SES, 83-92

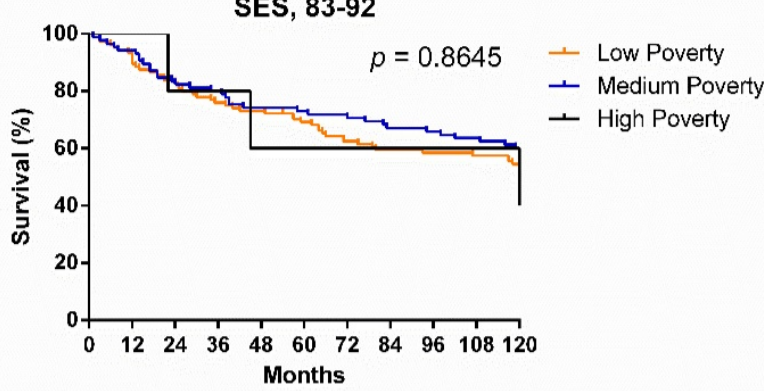

SES, 93-02

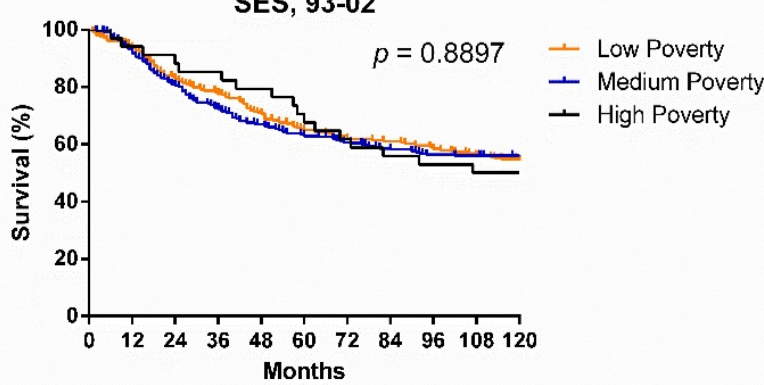

SES, 03-12

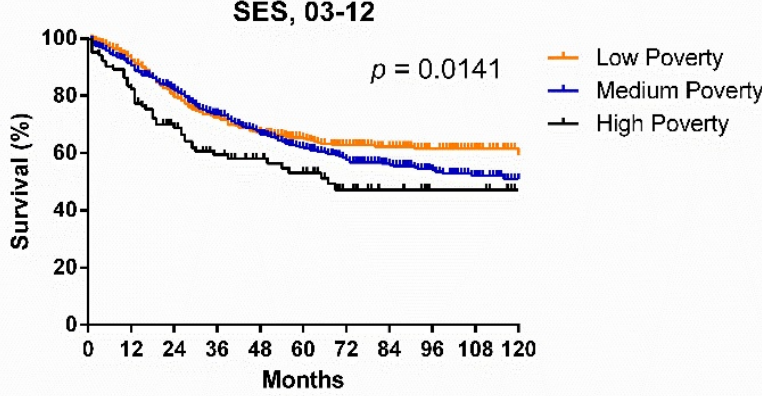

Figure 4. Kaplan-Meier survival analysis according to race and SES for patients with SyS at 18 SEER sites across three decades and in each decade. 
As illustrated in Figure 8, the percentage of young patients aged 0-14 and 15-29 years increased, whereas the percentage of old patients aged 45-59 and $60+$ years decreased, over the three decades. A decreasing percentage of localized SyS was observed, but an increasing percentage of regional and distant
SyS. In addition, the percentage of SyS in the extremities decreased, whereas the percentage of SyS in the trunk and lungs and pleura increased. Pathologically, the percentage of NOS SyS decreased dramatically, with a robust increase in the percentage of spindle SyS. a
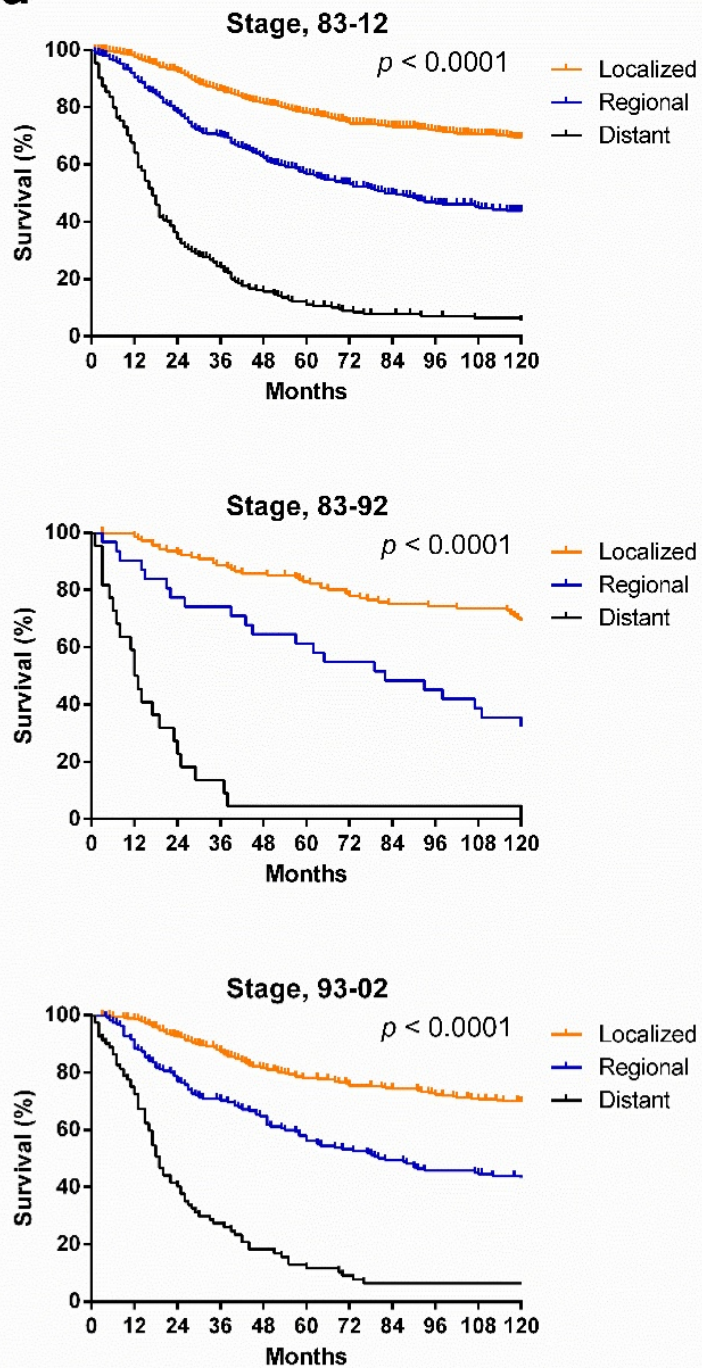

Stage, 03-12

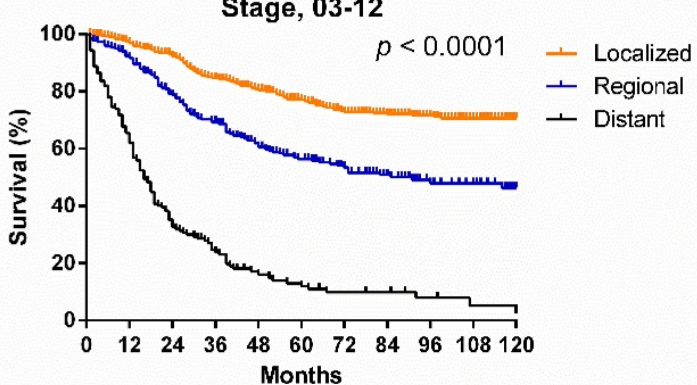

b

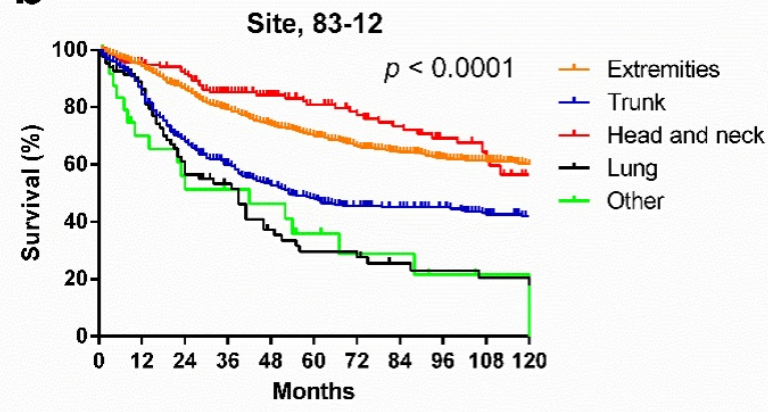

Site, 83-92

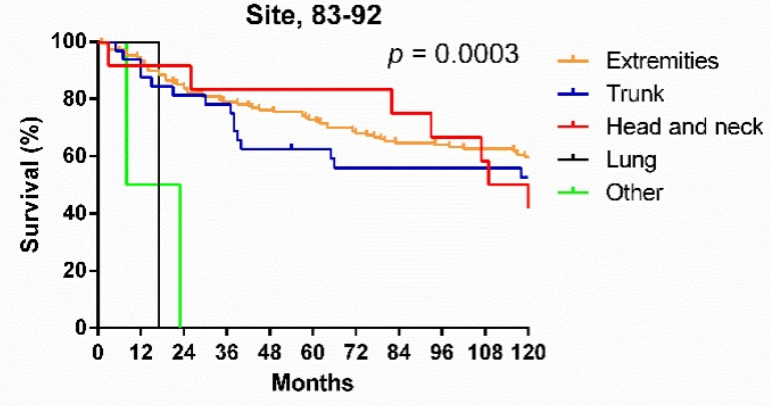

Site, 93-02

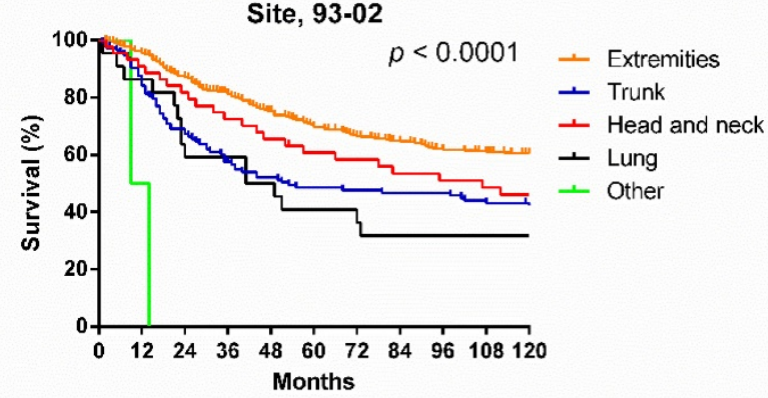

Site, 93-02

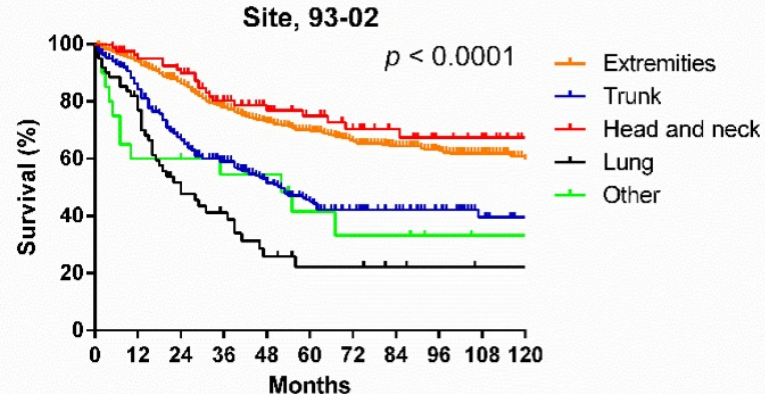

Figure 5. Kaplan-Meier survival analysis according to stage and site for patients with SyS at 18 SEER sites across three decades and in each decade. 

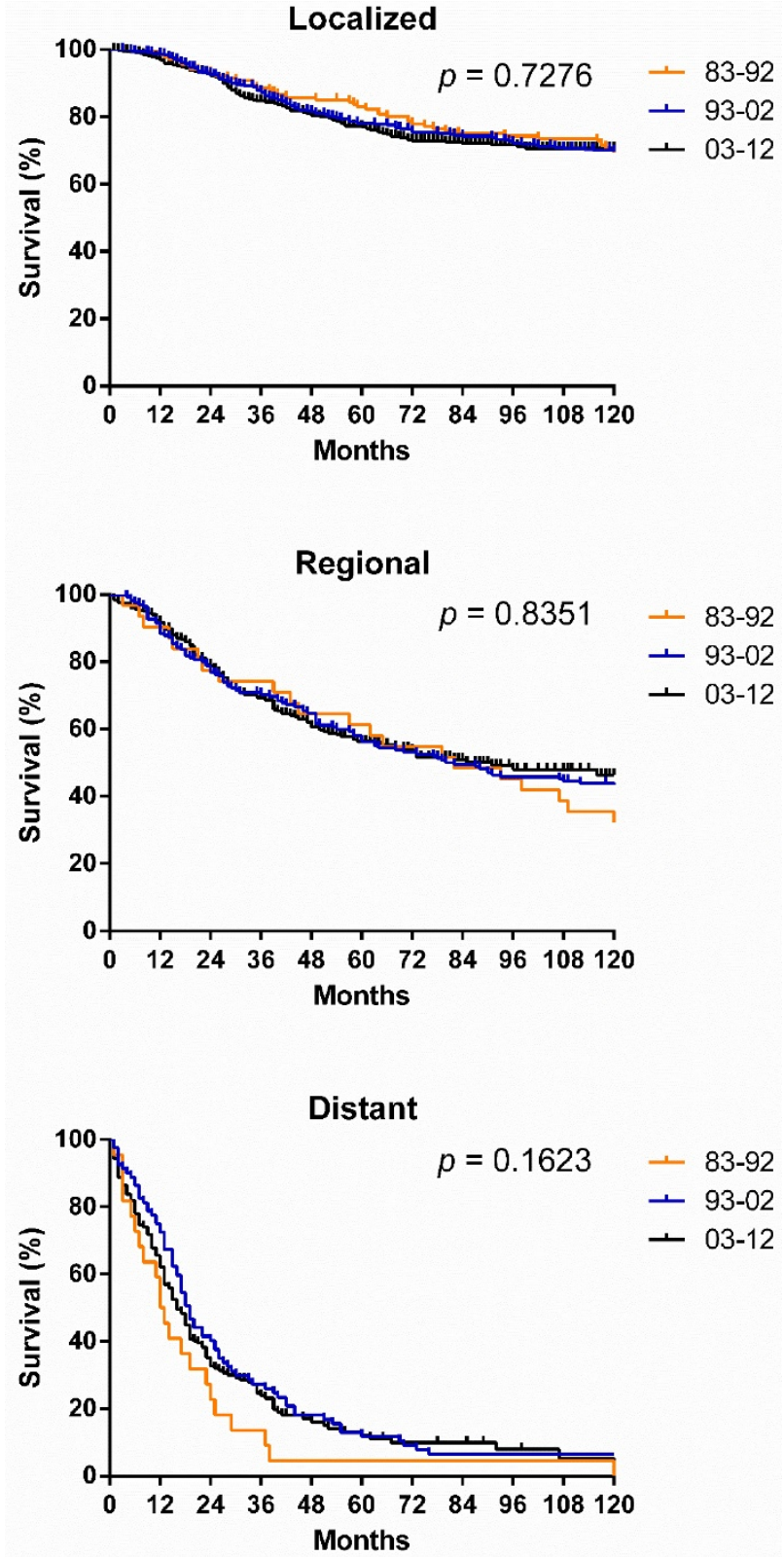

Figure 6. Kaplan-Meier survival analysis according to stage for patients with SyS at 18 SEER sites across three decades.

\section{Discussion}

This is the first study of incidence and survival in SyS based on a large population in the United States to date. We demonstrated the increasing incidence of SyS in the total population and in each age group except for the 0-14 years group in the three decades. However, no survival improvement in SyS was observed in the three decades, indicating the pressing need for the development of novel and effective treatments.

One widely believed cause for SyS is a chromosome abnormality known as $\mathrm{t}(\mathrm{X} ; 18)$ (p11.2; q11.2), resulting in the formation of SS18-SSX fusion oncogenes. It can be seen in more than $90 \%$ of SyS cases and therefore is believed to be the cause of SyS [10]. But the real etiological factors, including cell of origin, remain unknown. The peak age of SyS occurrence was 15-29 years, which differed from previous reports, in which the majority of SyS patients were aged 15 to 40 years [11]. The unimproved clinical outcome across the three decades may be partly attributed to the aging patient population. The incidence at each stage or site of origin increased, with robust increase noted in the localized cases and in cases originating in the extremities, respectively. The second most frequent site in each decade was the trunk, differing from previous studies in which the head and neck region was believed to be the second most frequent site [12].

Survival in SyS patients did not significantly change across the three decades, but of interest, survival in 1983-1992 was slightly longer than in 1993-2002, which showed a survival advantage compared with 2003-2012. The shift in patient clinical characteristics was responsible for the unchanged survival. First, the patient population was aging due to the decreasing percentage of younger patients and increasing percentage of older patients across the three decades. Second, the percentage of patients with regional or distant SyS and a worse prognosis increased compared with the percentage of patients with localized SyS. Third, the decreasing percentage of cases in the extremities and increasing percentage of cases in the trunk and lung and pleura contributed to the unimproved treatment outcomes.

In terms of sex, females showed survival advantage, and the survival gaps between the sexes widened across the three decades, with narrowing $p$ values, suggesting that the biological differences between the sexes may play an increasingly important role in the pathogenesis of SyS. In terms of stage, survival time of localized, regional, and distant cases differed significantly, with the longest survival in localized cases in each decade. However, no significant survival improvement was shown in localized, regional, or distant cases in the three decades combined. Although survival for patients of different races did not significantly differ, the gaps among them widened as illustrated by decreasing $p$ values. Similar results were observed when patients were stratified by SES and significant survival difference among different SES groups was observed in the third decade.

Although histologic subtypes were not reported to have a significant impact on clinical outcomes [13], in the present analysis, after stratification according to different pathological types, survival time for patients with various pathological types differed significantly 
in 1983-2012, with the best prognosis in patients with biphasic SyS. Also, survival gaps among them widened each decade, with decreasing $p$ values. However, survival for patients with each pathological type alone did not improve significantly across the three decades. The decrease in survival for NOS SyS may be partly attributed to the following facts. First,

a
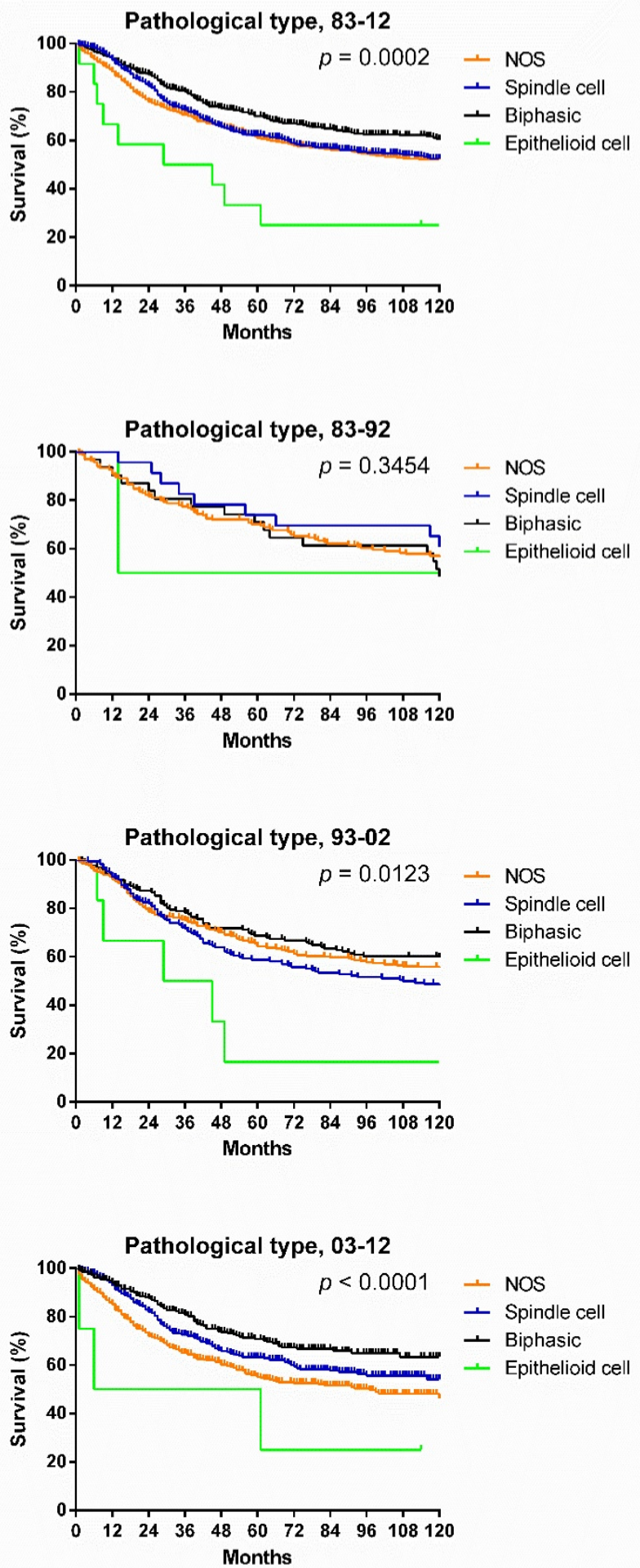

the other pathological types mixed in the NOS were more effectively excluded from the NOS in the last two decades, as illustrated by the decreasing percentage of NOS, due to improvement in pathological diagnosis. Second, the other pathological types, mainly biphasic SyS, showed a better prognosis than NOS SyS did.

b
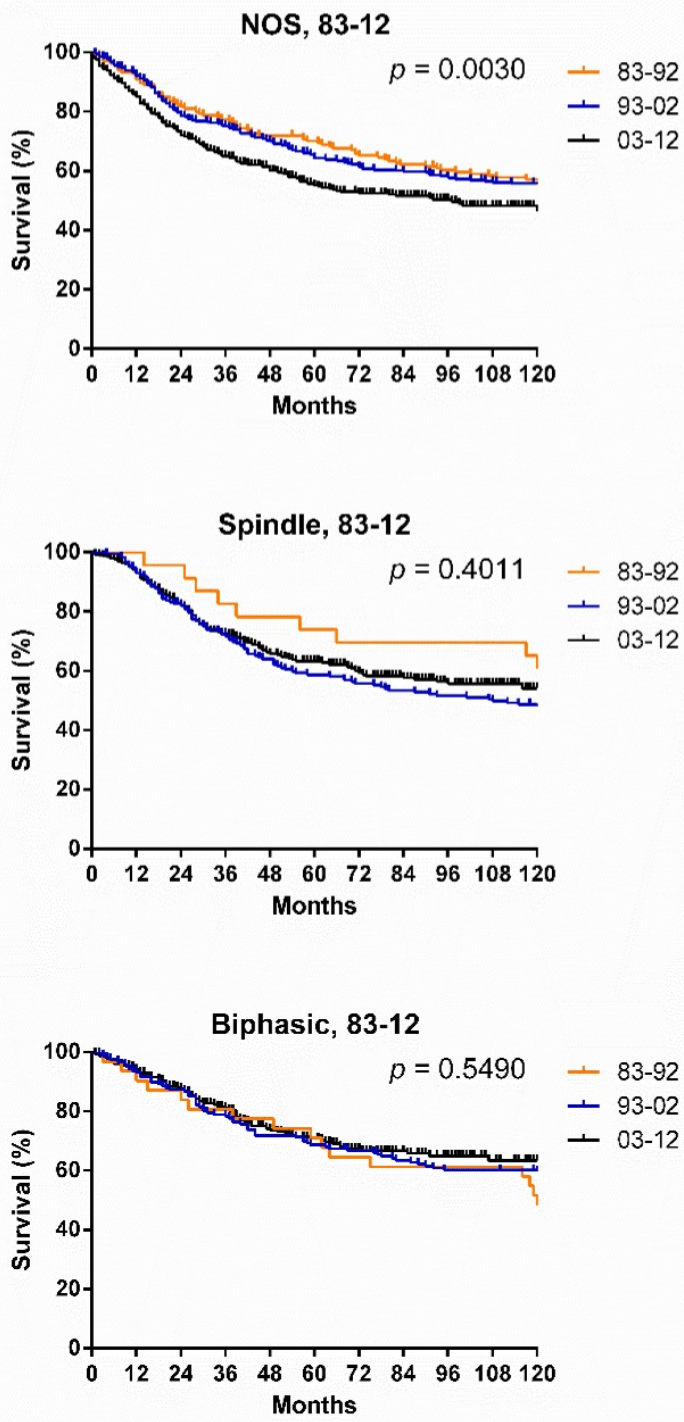

Epithelioid, 83-12

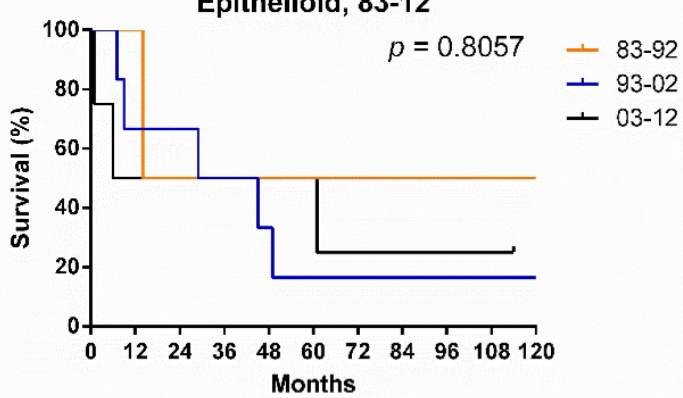

Figure 7. Kaplan-Meier survival analysis of pathological types according to calendar period (a) and pathological types (b) for patients with SyS at 18 SEER sites across three decades and in each decade. 
a

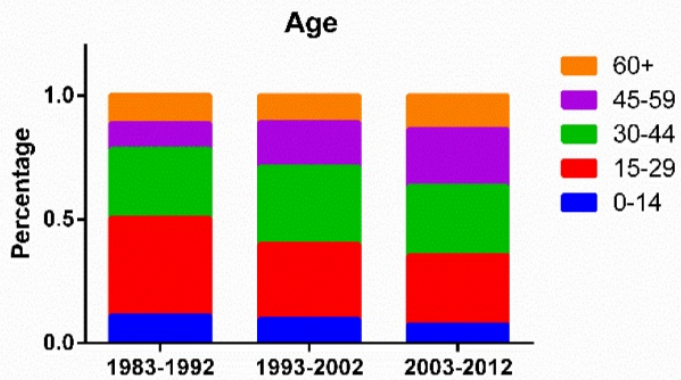

b

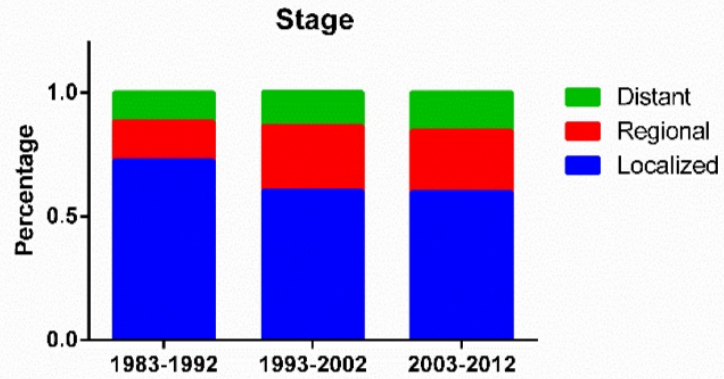

C

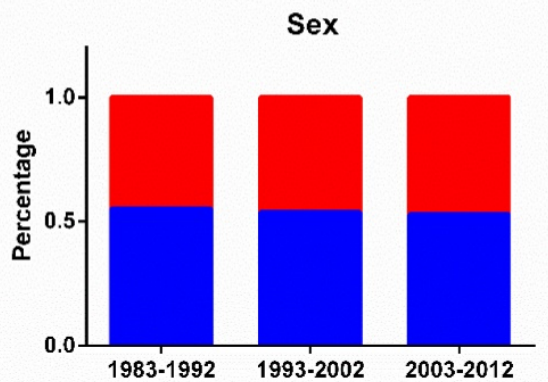

d

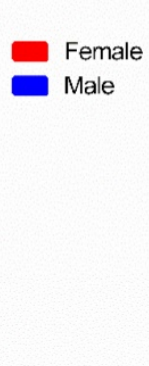

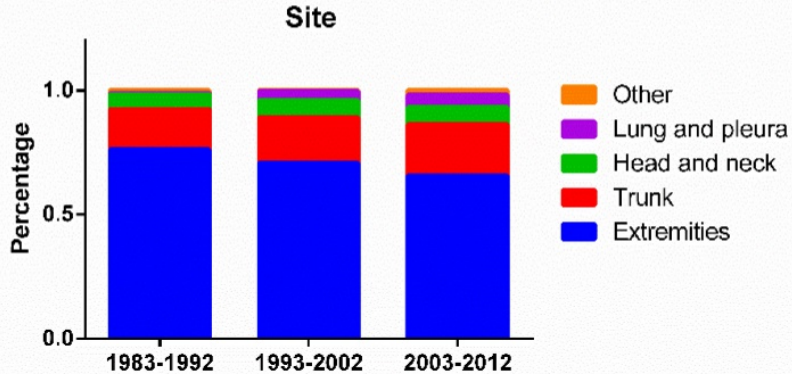

f

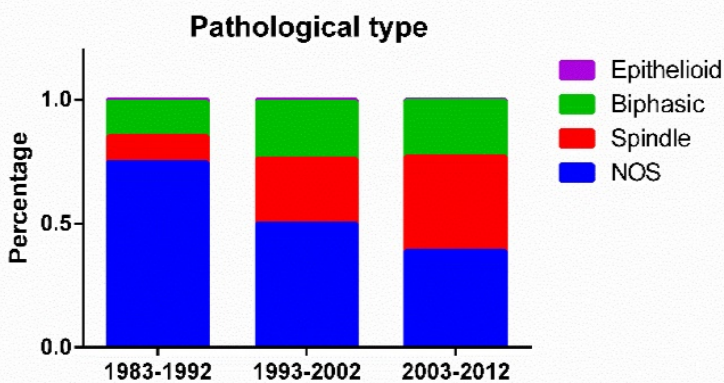

Figure 8. Changes of the percentage of patients in age (a), stage (b), sex (c), site (d), race (e), and pathological types (f) across three decades.

Treatments for SyS have been developed, such as VEGF targeting antiangiogenic therapy. VEGF targeting therapy functions by blocking the signaling of VEGF that depends on the chromosomal translocation SS18-SSX [14]. Cellular therapy targeting NY-ESO-1 has also been proven effective in synovial sarcoma [15]. Pazopanib has been used as a second-line therapy in SyS [16]. Looking ahead, there are many potential targets for SyS in the era of precision medicine. Potential molecular targets include the SS18-SSX fusion protein and overexpressed proteins such as bcl-2, EGFR, and HER-2/neu [17-19].
It is noteworthy that the results and conclusions here were based on the incidence and survival data in selected areas, and therefore it should be interpreted with cautions while applying to other regions. In addition, the internal under-registration and misclassification of SyS inside SEER database may be the potential sources of error and bias $[7,8]$.

Overall, our study showed the increasing incidence of SyS and more importantly, the unimproved survival between 1983 and 2012. Analyzing the incidence and survival trends of SyS in these last three decades may aid in predicting future trends of incidence and survival. Increasing incidence 
of SyS highlights the urgency for improving awareness in diagnosing SyS. In addition, it is of great importance to improve clinical management of SyS by developing novel treatments through studies clarifying the tumorigenesis and thereby determining potential therapeutic targets in SyS.

\section{Supplementary Material}

Supplementary Table S1.

http://www.jcancer.org/v08p1759s1.pdf

\section{Acknowledgments}

This research was supported by the National Natural Science Foundation of China (81500030) and the Natural Science Foundation of Guangdong Province (2016A030313272, 2016A030313277).

\section{Competing Interests}

The authors declare no competing financial interests.

\section{References}

1. Rajwanshi A, Srinivas R, Upasana G. Malignant small round cell tumors. J Cytol. 2009; 26: 1-10.

2. Mallen-St Clair J, Arshi A, Abemayor E, St John M. Factors Associated With Survival in Patients With Synovial Cell Sarcoma of the Head and Neck: An Analysis of 167 Cases Using the SEER (Surveillance, Epidemiology, and End Results) Database. JAMA Otolaryngol Head Neck Surg. 2016

3. Cui Q, Li D, Liu S, Jiang W, Guo J, Liu C, et al. Clinical report of intra-arterial interventional chemotherapy for synovial sarcoma on limbs. J Cancer Res Ther. 2016; 12: 73-6.

4. Radhakrishnan V, Dhanushkodi M, Narayanswamy K, Raja A, Sundersingh S, Sagar T. Synovial sarcoma of kidney in a child: A rare presentation. J Indian Assoc Pediatr Surg. 2016; 21: 75-7.

5. Madabhavi I, Patel A, Anand A, Panchal H, Parikh S. Primary Mediastinal Synovial Sarcoma with subsequent development of Primary Adenoid Cystic Carcinoma of Lung presenting as superior vena cava syndrome. Clin Respir J. 2016.

6. Sultan I, Rodriguez-Galindo C, Saab R, Yasir S, Casanova M, Ferrari A. Comparing children and adults with synovial sarcoma in the Surveillance, Epidemiology, and End Results program, 1983 to 2005: an analysis of 1268 patients. Cancer. 2009; 115: 3537-47.

7. Krieger N, Chen JT, Waterman PD, Rehkopf DH, Subramanian SV. Race/ethnicity, gender, and monitoring socioeconomic gradients in health: a comparison of area-based socioeconomic measures--the public health disparities geocoding project. Am J Public Health. 2003; 93: 1655-71.

8. Krieger N, Chen JT, Waterman PD, Soobader MJ, Subramanian SV, Carson R. Geocoding and monitoring of US socioeconomic inequalities in mortality and cancer incidence: does the choice of area-based measure and geographic level matter?: the Public Health Disparities Geocoding Project. Am J Epidemiol. 2002; 156: 471-82

9. Sun H, Ma H, Hong G, Sun H, Wang J. Survival improvement in patients with pancreatic cancer by decade: a period analysis of the SEER database, 1981-2010. Sci Rep. 2014; 4: 6747.

10. Tamaki S, Fukuta M, Sekiguchi K, Jin $Y$, Nagata S, Hayakawa K, et al SS18-SSX, the Oncogenic Fusion Protein in Synovial Sarcoma, Is a Cellular Context-Dependent Epigenetic Modifier. PLoS One. 2015; 10: e0142991.

11. Thway K, Fisher C. Synovial sarcoma: defining features and diagnostic evolution. Ann Diagn Pathol. 2014; 18: 369-80.

12. Al-Daraji W, Lasota J, Foss R, Miettinen M. Synovial sarcoma involving the head: analysis of 36 cases with predilection to the parotid and temporal regions. Am J Surg Pathol. 2009; 33: 1494-503.

13. el-Naggar AK, Ayala AG, Abdul-Karim FW, McLemore D, Ballance WW, Garnsey L, et al. Synovial sarcoma. A DNA flow cytometric study. Cancer. 1990; 65: 2295-300.

14. Wakamatsu T, Naka N, Sasagawa S, Tanaka T, Takenaka S, Araki N, et al. Deflection of vascular endothelial growth factor action by SS18-SSX and composite vascular endothelial growth factor- and chemokine (C-X-C motif) receptor 4-targeted therapy in synovial sarcoma. Cancer Sci. 2014; 105: 1124-34

15. Robbins PF, Morgan RA, Feldman SA, Yang JC, Sherry RM, Dudley ME, et al. Tumor regression in patients with metastatic synovial cell sarcoma and melanoma using genetically engineered lymphocytes reactive with NY-ESO-1. J Clin Oncol. 2011; 29: 917-24

16. Sugitani A, Asai K, Kojima K, Eguchi Y, Kawaguchi T, Ohsawa M, et al. Primary Pleural Synovial Sarcoma Treated with Pazopanib. Intern Med. 2015; 54: 2051-5.

17. Albritton KH, Randall RL. Prospects for targeted therapy of synovial sarcoma. J Pediatr Hematol Oncol. 2005; 27: 219-22.

18. Thomas DG, Giordano TJ, Sanders D, Biermann S, Sondak VK, Trent JC, et al. Expression of receptor tyrosine kinases epidermal growth factor receptor and HER-2/neu in synovial sarcoma. Cancer. 2005; 103: 830-8.

19. Teng HW, Wang HW, Chen WM, Chao TC, Hsieh YY, Hsih $\mathrm{CH}$, et al. Prevalence and prognostic influence of genomic changes of EGFR pathway markers in synovial sarcoma. J Surg Oncol. 2011; 103: 773-81. 